\title{
The Development of Theoretical Basis of Mathe- matical Model of Crushed Rock Particle-Particle Packing
}

\author{
Sergey Shabaev ${ }^{1, *}$, Sergey Ivanov ${ }^{1}$, and Martel Nikita ${ }^{1}$ \\ ${ }^{1}$ T. F. Gorbachev Kuzbass State Technical University, 650000, Kemerovo, Russian Federation
}

\begin{abstract}
The existing mathematical models that predict the poly-fractional granular media packing density do not take into account their packing degree, which gives a fairly large deviation of the simulation results from the actual experimental values (up to $20-25 \%$ ). To increase the reliability of prediction of the elastic modulus of crushed rocks, it is necessary to develop the mathematical model that would give more adequate values, since it is known that a change in the granular medium packing density by only $5-10 \%$ can lead to a change in the elastic modulus up to 1.5-2 times. The conditions under which smaller particles can be placed in the free space formed after packing larger particles are determined in this work on the basis of the performed computer simulation. It is established that particles of the $i$-th component of crushed rock can be placed in the free space formed after packing all the previous (larger) components, if the free space volume exceeds the ratio of the actual volume of the particles of the considered component to the packing density of onedimensional particles to a degree that is exponential function of the serial number of the component.
\end{abstract}

\section{Introduction}

The performance of open-cut vehicles is significantly impacted, among other factors, by the quality of roads $[1,2]$. And, despite the fact that recently more and more research are carried out on asphalt and cement concrete road topping, as well as on increasing their bearing capacity using geosynthetic materials [3-5], yet their largest share is still occupied by nonmetallic structures $[6,7]$.

In addition to standard construction materials, crushed overburden and carbon-dispersed crushed rocks can be used to construct pavement layers of temporary open-cut roads. To predict the packing degree, as well as deformation and strength characteristics, it is necessary to know their bulk density, which in turn depends on their particle size distribution and the compaction effect values.

Today, apart from mathematical models devoted to the packing of one-dimensional particles [8-11 and others], there are mathematical models that predict the bulk mass (density) of anisometric materials using phenomenological and fine-structural approaches. However, the main disadvantage of all these models is that they do not take into account the packing degree of the granular material, and therefore it is necessary to develop the mathematical model that allows

${ }^{*}$ Corresponding author: shabaev81@rambler.ru 
determining the packing density of crushed rock particles not only taking into account their particle size distribution, but also the compaction effect values.

\section{Materials and methods}

When considering the granular material packing, assumptions are always made. All previously developed mathematical models for the packing of both single-dimensional and multidimensional particles imply that they have the form of a cube, a tetrahedron, an octahedron, a sphere, and others. In reality, the particle shape of the granular material is so diverse and not repeatable that the probability of any regular shaping is very small, regardless of its type. In this regard, it was assumed that the shape of all particles is spherical. In addition, it is also assumed that all the largest particles that make up the granular material are evenly distributed throughout its volume, that is, the segregation phenomenon is not taken into account. When modeling the packing density this allows considering not the entire set of particles, but only one largest particle and a part of the space adjacent to it.

To develop the mathematical model capable to predict the crushed rock volume mass (density), it is necessary to know the greatest packing density of anisometric particles. To solve the problem, we assume that the crushed rock consists of $N$ components (a specific fraction of crushed rock is taken for each component), each of which is characterized by an average $D_{i}$ particle diameter, and the largest component (\#1) has an average $D_{\max }$ particle size. The average particle size of each component is determined from the dependency (making another assumption that the average particle size of each subsequent component is 2 times smaller than that of the previous one):

$$
\frac{D_{\max }}{D_{i}}=2^{i-1}
$$

where $i$ - the component number.

Assuming that the largest particles are evenly distributed throughout the volume, we will consider only one such particle with an adjacent part of the space, which is a limited discrete structure, the set of which makes up the crushed rock macrostructure. For this, two threedimensional arrays including the number of points (nodes) sufficient for discretization of the smallest particles that make up the crushed rock, with the value of each point equal to zero, are created in the virtual memory of computer:

$$
\operatorname{Massive} 1(X, Y, Z)=0, \text { Massive } 2(X, Y, Z)=0,
$$

where $X, Y, Z$ - the number of points in the array along the axes $x, y, z$ respectively.

Then, the largest component is inscribed into the central part of the first array area by activating zeroed points (giving them a unit value):

$$
\begin{aligned}
& X=\left(\frac{X}{2}-\frac{X}{2 \cdot K x y z}\right),\left(\frac{X}{2}-\frac{X}{2 \cdot K x y z}\right)+1, \ldots,\left(\frac{X}{2}+\frac{X}{2 \cdot K x y z}\right) \\
& y \min =\frac{X}{2}-0,5 \cdot \sqrt{-4 \cdot x^{2}+8 \cdot x \cdot \frac{X}{2}-4 \cdot\left(\frac{X}{2}\right)^{2}+\left(\frac{X}{K x y z}\right)^{2}} ; \\
& y \max =\frac{X}{2}+0,5 \cdot \sqrt{-4 \cdot x^{2}+8 \cdot x \cdot \frac{X}{2}-4 \cdot\left(\frac{X}{2}\right)^{2}+\left(\frac{X}{K x y z}\right)^{2}} ;
\end{aligned}
$$




$$
\begin{gathered}
z \min =\frac{Z}{2}-0,5 \cdot \sqrt{-4 \cdot x^{2}+8 \cdot x \cdot \frac{X}{2}-4 \cdot\left(\frac{X}{2}\right)^{2}-4 \cdot y^{2}+8 \cdot y \cdot \frac{Y}{2}-4 \cdot\left(\frac{Y}{2}\right)^{2}+\left(\frac{Y}{K x y z}\right)^{2}} ; \\
z \max =\frac{Z}{2}+0,5 \cdot \sqrt{-4 \cdot x^{2}+8 \cdot x \cdot \frac{X}{2}-4 \cdot\left(\frac{X}{2}\right)^{2}-4 \cdot y^{2}+8 \cdot y \cdot \frac{Y}{2}-4 \cdot\left(\frac{Y}{2}\right)^{2}+\left(\frac{Y}{K x y z}\right)^{2}} ; \\
Z=z \min , z \min +1, \ldots, z \max ; \\
\text { Massive }(X, Y, Z)=1,
\end{gathered}
$$

where $K x y z-$ multiplicative factor equal to:

$$
K x y z=K x \cdot K y \cdot K z
$$

$K x, K y, K z$ - coefficients taking into account the distances between particles along axes $x, y, z$ respectively; those being equal to one when the distance between the centers of the two balls is equal to $D_{\max }$.

Assuming that the volume of a single discrete structure is equal to one, and the distances between centers of two balls along the $\mathrm{x}, \mathrm{y}, \mathrm{z}$ axes are equal to each other, the volume occupied by the largest particle is equal to:

$$
V=C_{u p}=\frac{\pi}{6 \cdot K x y z^{3}}
$$

where $C_{u p}$ - the packing density of the largest particles, numerically equal to the volume occupied by the largest particle in a single discrete structure of the material.

The results of previously performed practical experiments showed that the packing density of one-dimensional particles of crushed rock, depending on the compaction effect values, ranges from 0.587 to 0.623 . In this regard, we assume that the packing density of the largest particles is 0.6 . Then we have:

$$
K x y z=\sqrt[3]{\frac{\pi}{6 \cdot C_{u p}}}=0.956 .
$$

The largest component having been inscribed in the first array; the location of the component \#2 center is randomly selected:

$$
\left\{\begin{array}{l}
x c 2=\operatorname{Randomize}(x) \\
y c 2=\operatorname{Randomize}(y) \\
z c 2=\operatorname{Randomize}(z) .
\end{array}\right.
$$

The location of the center of the second component having been selected; all possible points of the array along the $\mathrm{x}, \mathrm{y}, \mathrm{z}$ axes are calculated, which will be occupied when inscribing this component, and the zero points of the second array are activated:

$$
\left\{\begin{array}{l}
x 2 \min =x c 2-D 2 / 2 \\
x 2 \max =x c 2+D 2 / 2
\end{array}\right.
$$




$$
\begin{gathered}
x 2=x 2 \min , x 2 \min +1, \ldots, x 2 \max ; \\
y 2 \min =y c 2-0,5 \cdot \sqrt{-4 \cdot x 2^{2}+8 \cdot x 2 \cdot x c 2-4 \cdot x c 2^{2}+D 2^{2}} ; \\
y 2 \max =y c 2+0,5 \cdot \sqrt{-4 \cdot x 2^{2}+8 \cdot x 2 \cdot x c 2-4 \cdot x c 2^{2}+(D 2)^{2}} ; \\
y 2=y 2 \min , y 2 \min +1, \ldots, y 2 \max ; \\
z 2 \min =z c 2-0,5 \cdot \sqrt{-4 \cdot x 2^{2}+8 \cdot x 2 \cdot x c 2-4 \cdot x 2^{2}-4 \cdot y 2^{2}+8 \cdot y 2 \cdot y c 2-4 \cdot y c 2^{2}+D 2^{2}} ; \\
z 2 \max =z c 2+0,5 \cdot \sqrt{-4 \cdot x 2^{2}+8 \cdot x 2 \cdot x c 2-4 \cdot x c 2^{2}-4 \cdot y 2^{2}+8 \cdot y 2 \cdot y c 2-4 \cdot y c 2^{2}+D 2^{2}} ; \\
z 2=z 2 \min , z 2 \min +1, \ldots, z 2 \max ; \\
\text { Massive } 2(x 2, y 2, z 2)=1 .
\end{gathered}
$$

If at least one of the activated points of the second array coincides with the activated points of the first array, all points of the second array are reset, and the new location of the center of the second component is selected. Otherwise, all coordinates of the activated points of the second array are transferred to the first array, and they are assigned the value of one, and the points of the second array are reset.

The algorithm allows inscribing component \# 2 several times, which allows inscribing the available free space of a single discrete structure as much as possible. After this, the procedure is repeated for component \#3 and so on for each subsequent component.

After packing each element, the proportion of the total number of points of the first array that have a non-zero value is calculated:

$$
A_{i}=\frac{\text { Massive1 }(X, Y, Z)=1 "}{\text { Massive1 }(X, Y, Z)=1 "+\text { Massivel }(X, Y, Z)=0 "} .
$$

Since this method considers a model that does not imply the obligatory contact of randomly arranged heavy spherical particles, that is, spherical particles that are randomly located not overlapping and not in contact with each other, multiple modeling is carried out to obtain the results adequate to the gravitational field. In this case, the final result is the case that provides the highest packing density of particles, since according to the research results [12-14], the packing density of spherical particles in a gravitational field exceeds the packing density of the same particles without taking it into account.

In previously performed studies, the hypothesis was assumed that a $D_{2}=0.5 \cdot D_{\max }$ size ball can be inscribed in the free space formed after packing larger balls, if its packing volume meets the condition:

$$
V_{2}^{u p}=\frac{V_{2}}{C_{u p}^{16-20 \cdot C_{u p}}} \leq V_{1}^{s v},
$$

where $V_{2}-$ the volume of the second component inscribed into the free space formed after packing the first component;

$V_{1}^{s v}$ - the volume of free space formed after packing the first component.

This hypothesis is proved only for the case of a two-component system, that is, when $i_{\max }=2$. Consequently, its development is required for a multicomponent system. In this re- 
gard, we accept the refined hypothesis, which consists in the fact that balls of smaller size can be inscribed in the free space formed after packing all larger balls, if their packing volume meets the condition:

$$
V_{i}^{u p}=\frac{V_{i}}{C_{u p}^{n_{i}}} \leq V_{i-1}^{s v},
$$

where $n_{i}$-exponent depending on the serial number of the $i$ component;

$V_{i-1}^{s v}$ - the volume of free space formed after packing all the components with sequence numbers $i-1, i-2, \ldots, 1$.

From the expression (26) it follows that the limiting (maximum) volume of the $i$-th component, which can be placed in the free space formed after the packing all the larger components, is achieved when the $n_{i}$ exponent is:

$$
\begin{gathered}
n_{i}=\log _{C_{u p}}\left(\frac{V_{i}}{V_{i-1}^{s v}}\right), \\
V_{i}=A_{i}-A_{i-1}, \\
V_{i-1}^{s v}=1-A_{i-1} .
\end{gathered}
$$

\section{Results}

In the course of multiple modeling, the exponent was determined corresponding to the highest

\begin{tabular}{|c|c|c|c|}
\hline $\begin{array}{c}\text { Component } \\
\text { number, } i\end{array}$ & $\begin{array}{l}\text { The ratio of the average particle } \\
\text { diameter of the largest (first) } \\
\text { component to the average parti- } \\
\text { cle diameter of the } i \text {-th compo- } \\
\text { nent, } D_{\max } / D_{i}\end{array}$ & $\begin{array}{l}\text { The proportion of the volume of } \\
\text { a single discrete structure occu- } \\
\text { pied by particles of components } \\
\text { with sequence numbers }(i, i-1, \ldots \text {, } \\
\text { 1), } A_{i}\end{array}$ & Exponent, $n_{i}$ \\
\hline 1 & 1 & 0.6000 & \\
\hline 2 & 2 & 0.6750 & 3.28 \\
\hline 3 & 4 & 0.7188 & 3.92 \\
\hline 4 & 8 & 0.7537 & 4.08 \\
\hline 5 & 16 & 0.7828 & 4.18 \\
\hline 6 & 32 & 0.8076 & 4.25 \\
\hline 7 & 64 & 0.8299 & 4.22 \\
\hline 8 & 128 & 0.8516 & 4.03 \\
\hline 9 & 256 & 0.8714 & 3.94 \\
\hline 10 & 512 & 0.8900 & 3.78 \\
\hline 11 & 1024 & 0.9062 & 3.75 \\
\hline 12 & 2048 & 0.9198 & 3.78 \\
\hline 13 & 4096 & 0.9325 & 3.61 \\
\hline 14 & 8192 & 0.9424 & 3.76 \\
\hline
\end{tabular}
packing density of anisometric particles for a given packing density of one-dimensional particles of $C_{u p}=0.60$ (table)

Table 1. The values of the exponent according to the results of computer simulation

The dependence of the exponent on the component number can be represented as a function (Figure): 


$$
n_{i}=0.5 \cdot \exp \left(-0.125 \cdot(i-6)^{2}\right)+3.75
$$

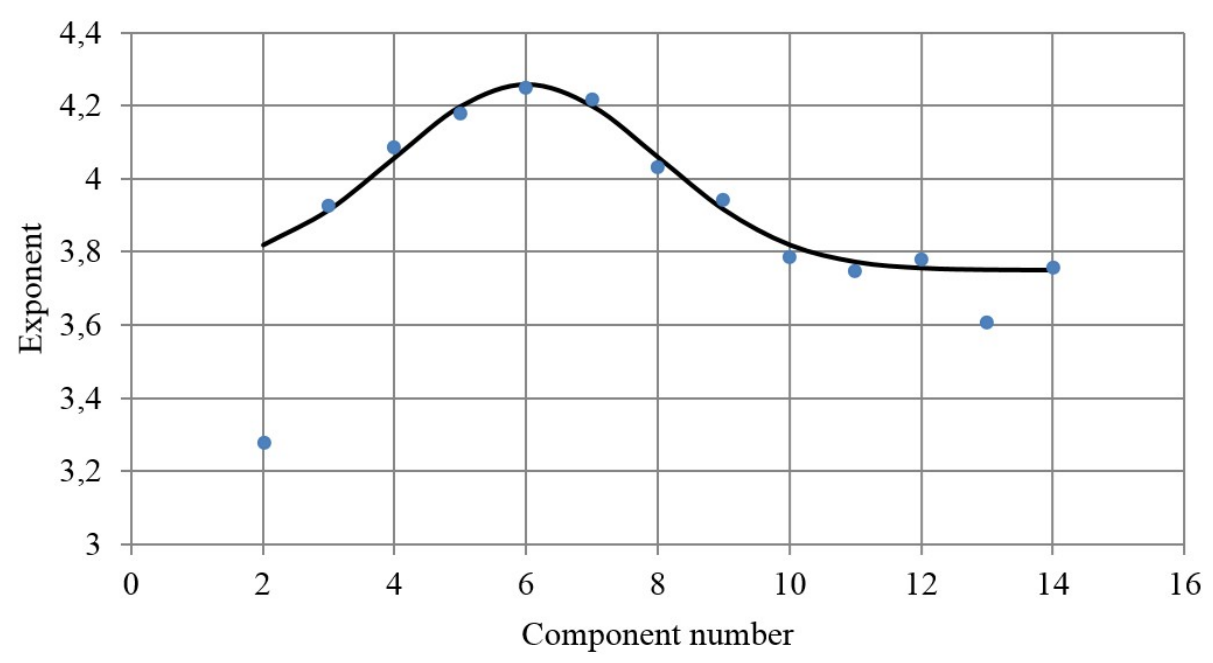

- Computer simulation data - Regression function

Fig. 1. Dependence of the exponent on the component number

Statistics data show that regression function (30) is adequate in relation to the results of computer simulation. If the component number $i=2$ is taken into consideration, the correlation ratio is 0.84 , the Fisher's variance ratio is 0.325 ; a critical value being 4.26 (significance level is 0.95). If the second component is not taken into account, the correlation ratio will be 0.977 , and the Fisher's variance ratio is 0.02 , which is certainly more adequate compared to the first option. Based on this, taking into account dependence (30), condition (26) may be written as:

$$
V_{i-1}^{s v} \geq \frac{V_{i}}{C_{u p}^{n_{i}}}, n_{i}=\left\{\begin{array}{l}
3.28, i=2, \\
0.5 \cdot \exp \left(-0.125 \cdot(i-6)^{2}\right)+3.75, i>2 .
\end{array}\right.
$$

Obtaining a relationship characterizing the limiting volume of crushed rock particles of a certain size, which can be placed in the free space formed after packing all the larger particles, is a prerequisite for developing a mathematical model of particle packing.

\section{Conclusions}

1. If all the particles that make up the crushed rock are divided into components (fractions) with an average particle size within each component equal to half of the average particle size of the previous component, then the component number is equal to the logarithm to base 2 of the ratio of maximum average particle size to average particle size of the considered component increased by one.

2. If the packing density of one-dimensional particles of crushed rock is taken equal to 0.6 , then the multiplicative coefficient, which is a product of partial coefficients that take into account the distance between the particles along the $3 \mathrm{D}$ space axes, is 0.956 .

3 . The $i$-th component particles of crushed rock can be placed in the free space formed after packing all the previous components, if the volume of free space exceeds the ratio of the actual volume of the particles of the component under consideration to the packing density of the sin- 
gle-dimensional particles to a degree representing an exponential function of the component serial number (except for the sequence number equal to 2).

\section{References}

1. Sara, L. Jackson, Geoforum, 66, 94 (2015)

2. B. Kinga, M. Goycoolea, A. Newman, European Journal of Operational Research, 257, 297 (2017)

3. A. A Khristoforova, M.D. Sokolova, S.E. Filippov, S.M. Semenova, I.N. Gogolev, Chemical technologies and nanomaterials, 41, 11 (2014)

4. A.A. Khristoforova, M.D. Sokolova, B. N. Zarovnyaev, A. N. Akishev, Mining Journal, 3, 47 (2016)

5. G. V. Solovyev K. I.Vatchnadze, Procedia Engineering, 189, 666 (2017)

6. D. V. Moldovan, Journal of Industrial Pollution Control., 33:1, 1007 (2017)

7. V. I. Chernobai, Journal of Coal Science and Engineering, 19:2, 219 (2013)

8. G. E. Mueller, Powder Technology, 203, 626 (2010)

9. M. M. Roozbahani, B. B. K. Huat, A. Asadi Powder Technology, 224, 46 (2012)

10. C. M. Wensrich, Powder Technology, 219, 118 (2012)

11. P. Jalali, M. Li, J. Chem. Phys., 120:2, 1138 (2004)

12. D. Cooper, Colloid and Interface Sch., 119:2, 442 (1987)

13. H. Solomon, H. Weiner, Commun. Statist. Theor. Meth., 2571, 111 (1986)

14. B. Radovskiy, H. Heckendorf, Wissenschaftliche Schriftenreihe der Technischen Hochschule (Gluckauf, Karl-Marx-Stadt, 171, 1975) 